\title{
An Advanced Treatise on Fixture Design and Planning
}




\title{
SERIES ON MANUFACTURING SYSTEMS AND TECHNOLOGY
}

Editors-in-Chief: Andrew Y. C. Nee (National University of Singapore, Singapore) J.-H. Chun (Massachusetts Institute of Technology, USA)

Assistant Editor: S. K. Ong (National University of Singapore, Singapore)

\author{
Published \\ Vol. 1: An Advanced Treatise on Fixture Design and Planning \\ Andrew Y. C. Nee, Z. J. Tao \& A. Senthil Kumar
}




\title{
An Advanced Treatise on
}

Fixture Design and Planning

\author{
Andrew Y. C. Nee \\ National University of Singapore \\ Z. J. Tao \\ Nanyang Polytechnic \\ A. Senthil Kumar \\ National University of Singapore
}




\section{Published by}

World Scientific Publishing Co. Pte. Ltd.

5 Toh Tuck Link, Singapore 596224

USA office: 27 Warren Street, Suite 401-402, Hackensack, NJ 07601

UK office: 57 Shelton Street, Covent Garden, London WC2H 9HE

\section{British Library Cataloguing-in-Publication Data}

A catalogue record for this book is available from the British Library.

\section{AN ADVANCED TREATISE ON FIXTURE DESIGN AND PLANNING}

Copyright $(\odot 2004$ by World Scientific Publishing Co. Pte. Ltd.

All rights reserved. This book, or parts thereof, may not be reproduced in any form or by any means, electronic or mechanical, including photocopying, recording or any information storage and retrieval system now known or to be invented, without written permission from the Publisher.

For photocopying of material in this volume, please pay a copying fee through the Copyright Clearance Center, Inc., 222 Rosewood Drive, Danvers, MA 01923, USA. In this case permission to photocopy is not required from the publisher.

ISBN 981-256-059-9

Editor: Tjan Kwang Wei 
A.Y.C. Nee

Z.J. Tao

A. Senthil kumar
To my wife Siew Kheng and daughters Joanne, Julianne, Suzanne for their patience and understanding

To my wife Hongbing for her patience and support

To my parents Prof Anantharajan and Ms Vijayalakshmi, and my wife Amutha and son Saravan for their help and understanding 
This page is intentionally left blank 


\section{Preface}

The primary goal of this book is to cover, as much as possible, the state-of-the-art development in the domain of computer-aided fixture design and planning. The text concentrates on key issues central to the development of computer-integrated manufacturing, such as fixture design automation, fixture clamping layout synthesis, clamping intensity optimisation, workpiece-fixture interaction, intelligent fixtures which are integrated with processing equipment or machine tools, Internetenabled fixture design and modular fixture database management.

This book is intended to be a reference text for academics, manufacturing and industrial engineers. It may also be used as a text for engineering graduate students in the discipline.

The organisation of the book is arranged in a topical manner. It begins with a concise presentation of the generic principles of fixture design in Chapter 1. Chapter 2 discusses the concept of integrated computer-aided fixture design system. It presents a 3D CAD-based system which is able to produce automated, interference-free fixture design and assembly solutions. Chapters 3 and 4 cover the framework and methodology to determine a viable clamping layout in terms of optimal clamping points, positive clamping actuation sequence, and minimal sustainable clamping intensities.

The dynamic interaction between a workpiece and its fixture plays a crucial role in fashioning the finished accuracy of a part. Experimental investigation presented in Chapter 5 provides an insight into the workpiece stability and the inherent dynamic nature of the workpiece-fixture system. The book then details the architecture of an intelligent fixturing system in Chapter 6, which is capable of tool path compensation and on-the-fly moderation of fixturing parameters so as to minimize workpiece displacement and distortion.

Chapter 7 presents a fixture element database management system which is essential to the automation of fixture design process. It is followed by a description of an Internet-based interactive fixture design system in Chapter 8. This system promotes the concept of collaborative design in a distributed manufacturing environment. 


\title{
Acknowledgements
}

During the period of preparing for the book, we have received excellent support from our colleagues. We would like to acknowledge the valuable contributions by Dr J.Y.H. Fuh, Dr M.A. Mannan and Dr Y.S. Wong at the National University of Singapore. Our graduate students have also contributed to this book with their research work, particularly: S. Senthilkumar, J.R. Dai, L. Lin, X.W. Gui, J. P. Sollie, Y.F. Wang, T.K. Xiao and Long Qin, and in specific chapters: Chapter $2-$ T.S. Kow; Chapter 7 - Y.M. Yip; and Chapter 8 - S.H Bok, Ratnapu Kiran Kumar, Sandeep Kumar Arya, Y.J. Ng and Fathianathan Mervyn. We would also like to thank K.W. Tjan at World Scientific for his support in the production of this book.

Last but not least, we would like to dedicate the book to three distinguished academicians who have contributed indirectly to this book, they are the late

Dr Ken Whybrew

Professor Amitabha Bhattacharyya

Professor Inyong Ham

Dr Ken Whybrew was the co-author of a first book "Advanced Fixture Design for FMS" written together with AYCN and ASK. Prof Bhattacharyya provided an unpublished manuscript written with Prof Ham to AYCN many years ago and some materials of this manuscript are presented in Chapter 1.

\author{
A.Y.C. Nee \\ National University of Singapore \\ Z.J. Tao \\ Nanyang Polytechnic, Singapore \\ A. Senthil kumar \\ National University of Singapore
}




\section{Table of Contents}

\section{Chapter 1 Introduction to Fixture Design}

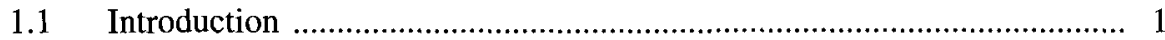

1.1.1 Elements of Fixtures ....................................................... 1

1.1.2 Importance of Fixtures in Manufacturing ............................... 3

1.1.3 General Requirements of a Fixture ......................................... 4

1.1.4 Fixture Design Fundamentals ............................................. 6

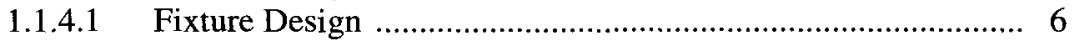

1.1.4.2 Fixture Design Criteria ………………............................... 8

1.1.4.3 Fixture Design Procedure ....................................................... 8

1.2 Locating Principles ........................................................................... 9

1.2.1 Introduction ...................................................................... 9

1.2.2 Restrictions on the Degrees of Freedom of a Workpiece ......... 9

1.3 Clamping Principles .................................................................... 10

1.3.1 Introduction ................................................................... 10

1.3.2 Basic Principles of Clamping .............................................. 11

1.3.2.1 Orientation of Locators vis-à-vis Clamping Force ................. 11

1.3.2.2 Effect of External Force on the Clamping Action .................. 11

1.3.2.3 Types of Clamps .............................................................. 12

$1.4 \quad$ Automation in Fixture Design ........................................................... 13

1.4.1 The Need for More Flexible Fixtures .................................. 13

1.4.2 Computer-aided Fixture Design Research ............................. 14

1.4.2.1 Geometric Analysis .......................................................... 14

1.4.2.2 Kinematic Analysis ......................................................... 15

1.4.2.3 Force Analysis .................................................................. 16

1.4.2.4 Deformation Analysis ..................................................... 17

1.4.3 Novel Clamping System Design ...................................... 17

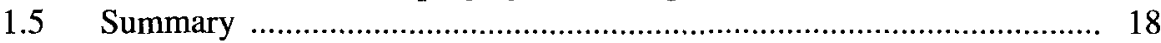

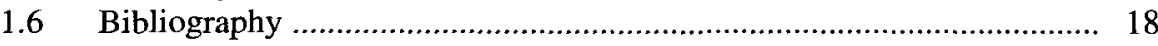

Chapter 2 Computer Aided Conceptual Fixture Design

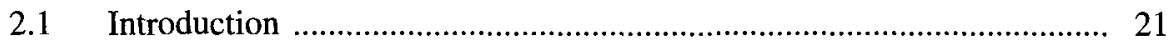

2.2 Integrated Prototype System ……................................................... 23 
2.2.1 Interactive Fixture Design System ..................................... 23

2.2.2 Automated Fixture Design ................................................. 27

2.3 Automated Fixturing Points Determination .......................................... 27

2.3.1 Generation of Candidate Supporting Points ......................... 29

2.3.2 Generation of Candidate Locating Points ............................ 30

2.3.3 Selection of Supporting Points .......................................... 31

2.3.4 Selection of Locating Points .............................................. 32

2.3.5 Selection of Clamping Points .............................................. 33

$2.4 \quad$ Machining Interference Detection ...................................................... 34

2.4.1 Cutter Location Source File ............................................. 34

2.4.2 Formation of Motion Silhouettes .......................................... 35

2.4.3 Generation of Cutter Swept Solid ........................................ 38

2.4.4 Collision Detection by Static Interference Check ................. 39

$2.5 \quad$ Illustrative Example ...................................................................... 40

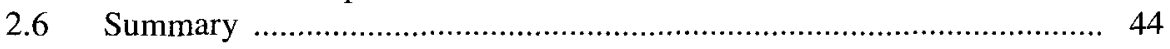

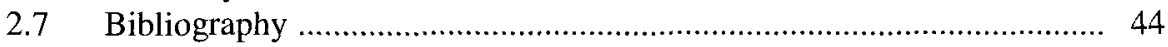

\section{Chapter $3 \quad$ Fixture Clamping Layout Synthesis}

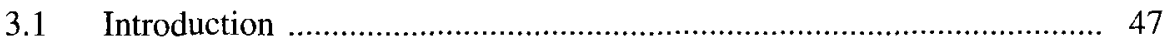

3.2 Importance of Analytical Approach ............................................... 48

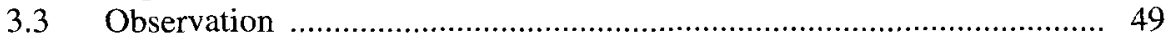

3.3.1 Clamping Equilibrium .................................................... 49

3.3.2 Force Closure …............................................................. 49

3.4 Contact Force Model .............................................................................. 49

3.4.1 Conservative Representation and Accuracy ......................... 52

3.4.2 Contacting Wrench .......................................................... 53

3.5 Gravitational Force ......................................................................... 54

3.6 Force Closure Condition .................................................................. 54

3.7 Clamping Equilibrium ……………………................................. 57

3.7.1 Grasping vs. Fixturing ……….......................................... 57

3.7.2 Condition for Clamping Equilibrium …….......................... 57

3.8 Verification of Force Closure for 2-D Workpiece ................................. 59

3.9 Criteria for Clamping Layout Reasoning ……..................................... 62

3.10 Automatic Generation of Clamping Points and Sequence ..................... 64

3.10.1 Consideration of Physical Constraints ................................... 66

3.11 Algorithm Implementation ........................................................... 66

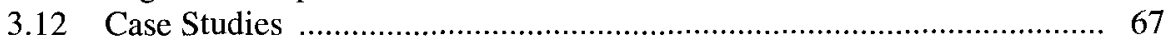

3.12.1 Planar Fixturing Problems .................................................. 67

3.12.1.1 Verification of Force Closure ................................................ 68

3.12.1.2 Best Clamping Points and Positive Clamping Sequence ........ 68

3.12.2 Spatial Fixturing Problems ………..................................... 73

3.12.2.1 Prismatic Workpiece ........................................................ 73

3.12.2.2 Engineering Workpiece .................................................. 76 


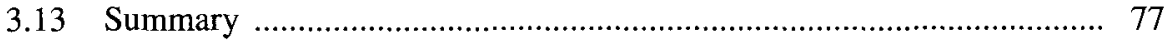

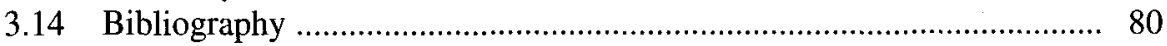

\section{Chapter 4 Optimisation of Dynamic Clamping Forces for a Fixture}

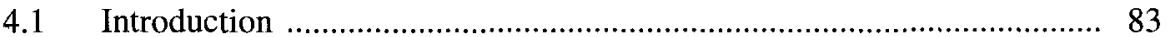

$4.2 \quad$ Direct Computation Approach …………………................................ 83

4.2.1 Minimal Clamping Force for Frictionless Fixturing .............. 83

4.2.2 An Illustrating Example ............................................... 85

4.2.3 Minimal Clamping Force for Frictional Fixturing …….......... 86

4.2.4 An Illustrating Example .................................................. 88

4.3 Generic Model for Clamping Force Optimisation ............................... 89

4.3.1 Complete Representation of Contact Force ........................... 89

4.3.2 Positive Location Constraint ................................................ 91

4.3.3 External Load .................................................................. 92

4.3.4 Workpiece Static Equilibrium ……….................................. 93

4.4 Optimal Clamping Force Algorithm ................................................. 94

4.4.1 Minimum Clamping ........................................................... 94

4.4.2 Safety Consideration ........................................................... 96

4.4.3 Maximum Clamping ........................................................... 96

4.4.4 Polygonal Contacts ............................................................ 98

4.5 Algorithm Implementation ……………........................................... 99

4.6 Case Studies ................................................................................. 101

4.6.1 Planar Fixturing ......................................................... 101

4.6.2 Spatial Fixturing ……….............................................. 103

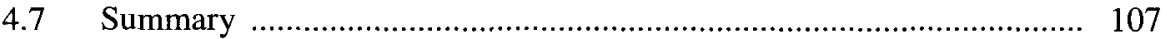

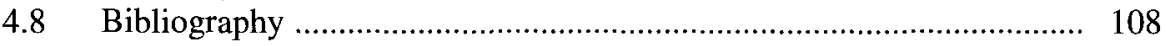

\section{Chapter 5 Workpiece-Fixture Interaction}

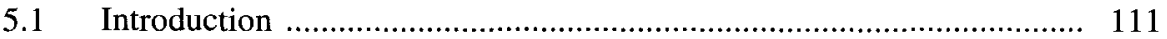

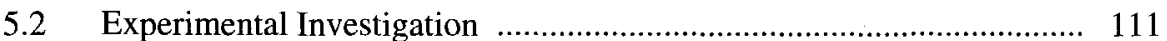

5.2.1 Sensor-integrated Fixture System ................................... 112

5.2.1.1 Forces within a Fixture .................................................. 112

5.2.1.2 Sensor Embedded Locator ................................................ 113

5.2.1.3 Data Acquisition for Reaction Forces .............................. 114

5.2.1.4 Physical Configuration .................................................. 115

5.2.2 Experimentation ......................................................... 116

5.2.2.1 Machining Operation ....................................................... 116

5.2.2.2 Top Clamping ………................................................. 118

5.2.2.3 Side Clamping ........................................................... 119

5.2.3 Discussion on Reaction Forces ....................................... 120

5.2.3.1 Top Clamping ......................................................... 122 
5.2.3.2 Side Clamping ............................................................. 122

5.2.3.3 Spectral Analysis of Reaction Forces ............................... 123

$5.3 \quad$ Finite Element Analysis ................................................................ 124

5.3.1 Contact Force/Deflection Relationships ............................ 127

5.3.2 Contact Approach ............................................................. 128

5.3.3 FEM Model and Solution ................................................ 129

5.3.4 Model Validation ............................................................ 129

5.3.4.1 Comparison of Reaction Forces ..................................... 130

5.3.5 Deformation under Dynamic Clamping ............................ 131

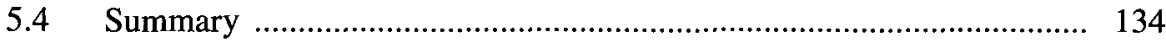

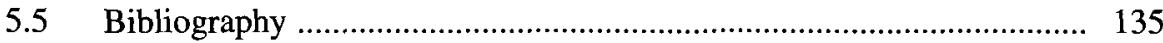

\section{Chapter 6 An Intelligent Fixturing System}

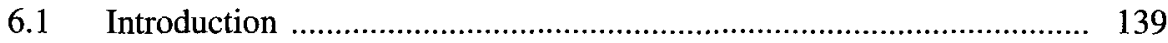

6.2 Architecture of an Intelligent Fixturing System ................................. 140

6.2.1 System Functions: From Viable Design to Optimal Execution

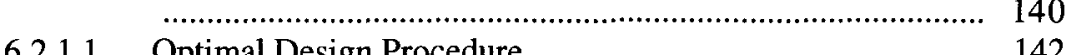

6.2.2 "Live" Fixture for Precision Machining

6.2.2.1 Clamping Sub-tasks ...................................................... 144

6.2.2.2 System Overview ........................................................... 144

6.3 Tool Path Compensation ……....................................................... 148

6.3.1 Determination of Workpiece Displacement ...................... 148

6.3.2 Workpiece Displacement by Experimental Measurement .... 151

6.3.3 Tool Path Compensation .................................................. 155

6.3.3.1 Linear Compensation .................................................... 155

6.3.3.2 Angular Compensation ................................................ 157

6.4 Dynamic Clamping Actuator ...................................................... 160

6.4.1 Fixturing Error Control Strategy ....................................... 160

6.4.2 Need for Dynamic Clamping Force ................................. 160

6.4.3 Dynamic Clamping Actuator ........................................... 161

6.4.3.1 Sensor Integration ......................................................... 161

6.4.3.2 Digital Interfacing ........................................................ 162

6.4.3.3 Clamping Force Control ............................................... 162

6.4.3.4 Clamping Task Programmability …................................. 162

6.5 Clamping Force Control Strategy .................................................... 162

6.5.1 DC Motor Servo Controller .............................................. 164

6.5.2 System Identification of Process ....................................... 165

6.5.3 Generalised Minimum Variance Model .............................. 166

6.6 Experimental Facility .................................................................... 168

$6.7 \quad$ Validation of Dynamic Clamping ........................................................ 169

6.7.1 Validating Experimentation and Discussions ..................... 171

6.7.2 Workpiece Stability under Dynamic Clamping ................... 173 
$6.8 \quad$ Impact of Intelligent Fixturing System ............................................ 175

6.8.1 Typical Dynamic Workholding Procedure .......................... 175

6.8.2 Experiments on Thin-walled Workpieces ............................ 176

6.8.3 Geometric Accuracy Improvement ……............................ 178

6.8.3.1 Comparison with Fixed Clamping ….................................. 179

6.8.4 Finished Surface Quality ................................................. 183

6.8.5 Process Cycle Time ...................................................... 184

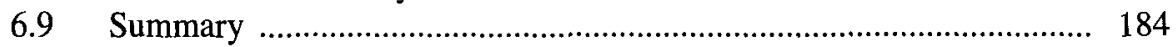

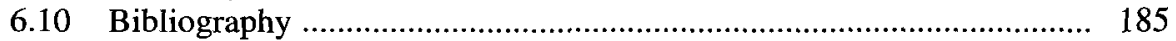

\section{Chapter 7 A Database Management System for Modular Fixtures}

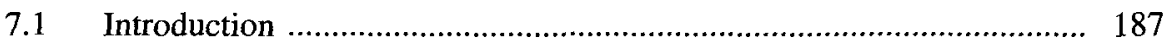

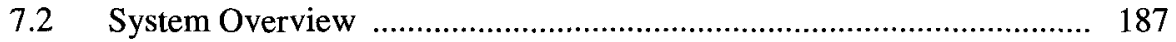

7.2.1 Enquiry Module ............................................................ 188

7.2.2 Element Database Module ................................................ 188

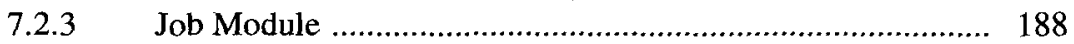

7.2.4 Schedule Module .......................................................... 188

7.2.5 Simulation Module ......................................................... 189

7.3 System Implementation .............................................................. 189

7.3.1 Data Storage Capability ................................................. 190

7.3.2 Data Access Capability ....................................................... 192

7.3.3 Data Sorting Capability ..................................................... 193

7.3.4 Simulation Capability …….............................................. 194

7.3.5 Data Extraction Capability ............................................ 195

7.3.5.1 Minimum Inventory Level ............................................... 195

7.3.5.2 Useful Life Span of Elements Expended ............................. 195

7.3.5.3 Number of Elements Used Per Month ............................... 196

7.3.5.4 Weight of Fixture Design (Without Workpiece) ................ 196

7.3.5.5 Cost of Fixture Design (Without Workpiece) ...................... 197

7.3.5.6 Percentage of Each of the Category of Element in a Job ...... 197

7.4 Evaluation of the FE_DBMS _.......................................................... 197

7.4.1 Inventory Enquiry ....................................................... 197

7.4.2 Cost Enquiry ............................................................... 198

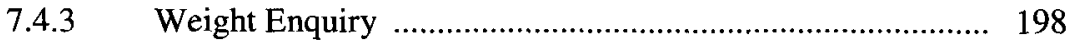

7.4.4 Monthly Enquiry ……….............................................. 199

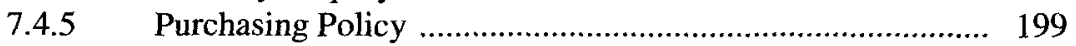

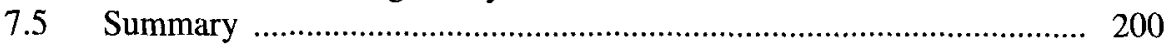

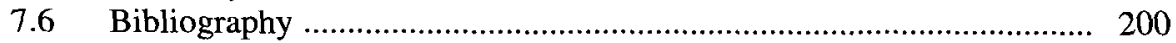


Chapter 8 An Internet-Enabled Smart Interactive Fixture Design System

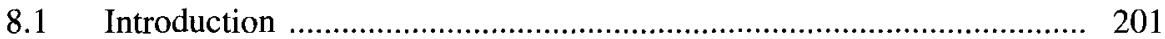

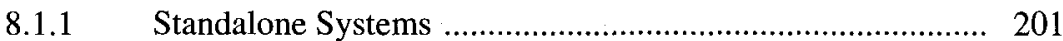

8.1.2 Internet-Enabled System ……....................................... 202

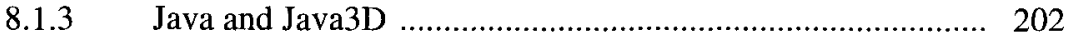

8.2 System Architecture .................................................................... 203

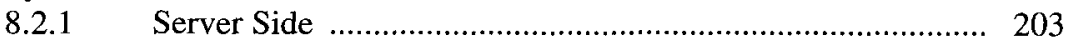

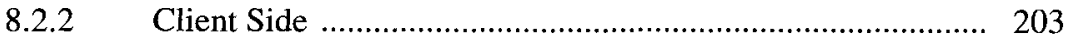

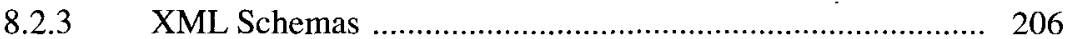

8.2.3.1 Fixture Design XML .................................................... 206

8.2.3.2 Facet Data XML .............................................................. 206

8.2.3.3 Product Data XML _........................................................ 206

8.2.4 System Application Process .............................................. 208

8.3 System Structure ........................................................................ 210

8.3.1 Application Process ...................................................... 210

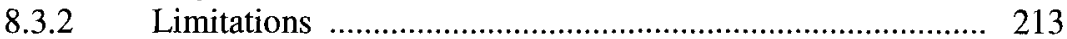

8.3.3 Storage Structure ........................................................ 213

8.3.4 Retrieval Structure ....................................................... 214

8.3.5 Changing a Design .................................................... 216

8.3.5.1 Object Delete Function ................................................... 216

8.3.5.2 Design Process for Existing System ................................. 218

$8.4 \quad$ System Architecture ................................................................ 220

8.4.1 Support Pin Rules ....................................................... 221

8.4.1.1 Support Rule Implementation Process ............................... 222

8.4.2 Locator Rules ............................................................ 222

8.4.2.1 Locator Rule Implementation Process ............................... 225

8.4.3 Clamp Rules ............................................................ 225

8.4.3.1 Side Clamp Rule Implementation Process .......................... 225

8.4.4 Limitations of SIFD Module ........................................... 228

8.5 An Illustrative Case Study ............................................................. 230

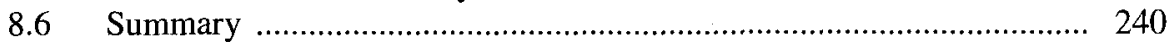

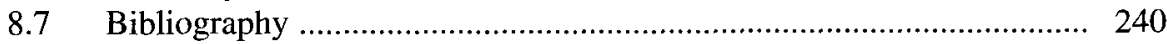

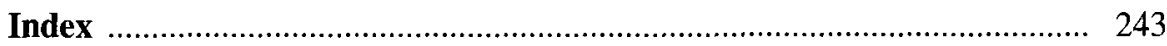

List of Authors 